\title{
The Living \& Breathing Study: a study of patients' views of asthma and its treatment
}

\author{
John Haughney ${ }^{\mathrm{a}, *}$, Greta Barnes ${ }^{\mathrm{b}}$, Martyn Partridge ${ }^{\mathrm{c}}$, Jennifer Cleland ${ }^{\mathrm{a}}$
}

\author{
a Department of General Practice and Primary Care, University of Aberdeen, Westburn Road, \\ Aberdeen AB25 2AY, UK \\ ${ }^{\mathrm{b}}$ Formerly National Asthma and Respiratory Training Centre, Warwick, UK \\ c Faculty of Medicine, NHLI Division, Charing Cross Hospital Campus, Imperial College, London, UK
}

\author{
KEYWORDS \\ Asthma; \\ Patient views; \\ Personal asthma action \\ plans; \\ Self-management; \\ Treatment
}

Summary Aim: To evaluate patient understanding of their asthma and determine patient preferences regarding the delivery of asthma care and treatment. Methods: Adults with asthma receiving treatment for mild to moderate asthma were recruited to a two-part study: a qualitative phase using a semi-structured interview schedule followed by a quantitative phase based on a structured interview schedule. All interviews were undertaken face-to-face. Setting and subjects: A random sample of 40 patients with mild to moderate asthma from seven areas of the UK took part in the qualitative phase of the study. In the quantitative phase, 517 patients on treatment for mild to moderate asthma were interviewed in person by market researchers. This population was achieved using a quota sampling approach that also achieved a representative demographic profile. Initial contact was made in door-to-door calls. Interviews took place in 64 locations across the UK Results: Ninety-one percent $(n=468)$ of respondents felt their asthma was under control, yet two-thirds $(n=339)$ experienced symptoms at least $2-3$ times a week. Only $24 \%(n=123)$ felt their asthma could improve over time, and $71 \%(n=366)$ received no advice from healthcare professionals on how their asthma might change in the future. Fourteen percent $(n=74)$ of respondents had no ongoing contact with any healthcare professional regarding their asthma. Fifty-eight percent $(n=301)$ were very satisfied with their asthma care, but this dropped to $33 \%(n=173)$ when respondents were shown asthma guidelines regarding what to expect from treatment. Sixty-two percent $(n=318)$ of respondents said their asthma varied at different times of the day, and $86 \%(n=444)$ stated that their asthma varied at different times of the year. Eighty percent $(n=414)$ of respondents had never been provided with a written, personal asthma action plan recommending changes patients could make themselves to prescribed treatment according to symptom severity, though $68 \%(n=353)$ said they would feel comfortable following such a plan. Conclusions: Most patients have low expectations of what can be achieved by asthma management and do not realise their condition can be improved. Many are resigned to the effects of poor asthma control until made aware that guidelines indicate this can be better. Given that many are receptive to the notion of written, personal asthma action plans, the implementation of these, supported by appropriate education, could help patients achieve improved asthma control. (c) 2003 General Practice Airways Group. Published by Elsevier Ltd. All rights reserved. 


\section{Introduction}

Asthma control, as measured against internationally agreed guidelines, is often suboptimal [1-4]. The Asthma Insights and Reality in Europe (AIRE) study [1] identified the current state of patient knowledge, attitudes and behaviour related to asthma in Europe, and suggested that asthma management fell far short of the goals set out in guidelines (Global Initiative for Asthma guidelines) [2]. The Asthma in Real Life (AIR) study [3] indicated that healthcare professionals underestimated the level of symptoms experienced by patients; for example, healthcare professionals estimated that less than $10 \%$ of patients had their ability to talk affected by asthma at least once a month, whereas almost $50 \%$ of patients reported this situation. The Needs of People with Asthma survey [4] also recorded a high level of morbidity, possibly associated with low expectation of care, or patients not sharing the same treatment goals or lifestyle objectives as healthcare professionals. These studies suggest that if outcomes for patients receiving asthma treatment are to improve, healthcare professionals should raise patient expectations by taking into consideration what matters to patients in terms of symptom control and then setting treatment goals accordingly. Advice, review and asthma management practice must be made available at times and in ways that are acceptable to patients who are managing a life-long regimen of medicine [5].

Meeting the needs of patients and improving communication between healthcare professionals and patients is likely to achieve better adherence to treatment [6,7]. Providing simple, written instructions, using a flexible approach, increases patient concordance [7]. Written, personalised action plans have been shown to improve health outcomes for people with asthma [7].

The British Thoracic Society/Scottish Intercollegiate Guidelines Network guidelines on asthma management recommend that the term 'personal asthma action plan' replaces 'self-management plan' and advise that these should be written and focus on individual needs, such as individual treatment goals [8].

Against this background, the Living \& Breathing Study aims to provide healthcare professionals with a detailed insight into what patients think about their asthma and its treatment. This information is likely to support healthcare professionals in developing a more flexible and personalised approach to asthma care.

\section{Methods}

\section{Phase 1 (qualitative)}

A qualitative study was undertaken in December 2000. Forty individual, in-depth interviews were carried out with asthma patients who met the British Thoracic Society (BTS) 1997 guidelines for Step 2 level of treatment (requiring and being prescribed 'reliever' and 'preventer' medication but no other asthma therapy). Patients were recruited from seven locations in the UK (North London, Sutton Coldfield, Reading, Stoneleigh, Dunstable, Lydd and Oldham) to ensure a range of geographical locations. A spread of ages, gender and socio-economic status was also achieved. Ten respondents from each of the following age groups: $14-18,19-30,31-50$ and 51-65 were recruited. There was an equal split of male to female respondents. Respondents were recruited to reflect the spectrum of socio-economic groups based on the market research occupational groupings: $A B s(n=4)$; C1s $(n=14)$; C2s $(n=14)$ and DEs $(n=8)$. Market researchers from an independent agency conducted a semi-structured, in-depth interview with each patient, exploring demographic details, the patients' asthma history, treatment, control and beliefs about their condition. The semi-structured interview schedule was developed by the authors and supported by members of an expert panel (see Acknowledgments). Questions were based on recent /evidence of patientknowledge and attitudes

\section{$[1,3]$.}

\section{Data analysis}

The interviews were recorded and transcribed, then analysed separately for emergent themes by two researchers. Emergent themes were discussed by the authors. JC examined the coded data independently for emergent themes and compared interpretations with $\mathrm{JH}$. Key findings were produced in the form of a report that detailed quotations from respondents and analysis under key headings, representing the emergent themes. This was independently examined by a panel of experts to ensure validation. The emergent themes were used to develop a questionnaire for the quantitative phase of the Living \& Breathing Study. This method was chosen to avoid creating a questionnaire based on the outcomes of previous studies. 


\section{Phase 2 (quantitative)}

Market researchers from an independent agency screened 2524 people from 64 individual sampling points across the UK in April 2001.

Respondents were contacted by door-to-door calls, including GP surgeries. Of these, $47 \%$ did not have asthma; $10 \%$ did not have time to participate or refused to participate and the asthma condition of a further $10 \%$ did not fulfil the inclusion criteria. The demographic detail of $13 \%$ did not fulfil the inclusion criteria.

A sample of 517 people currently undergoing treatment for mild to moderate, persistent asthma at Steps $1(n=254,49 \%) 2(n=196,38 \%)$ and 3 or more $(n=67,13 \%)$ of the BTS guidelines was selected. The sample was selected to achieve a representative, demographic profile as given further.

- Socio-economic status: AB (16\%); C1 (36\%); C2 (24\%); DE (24\%). Respondents were asked which of a group of occupations applied to them.

- Location: England (72\%); Wales (9\%); Scotland (9\%); N Ireland (10\%).

- Gender: Female (62\%); male (38\%).

- Age: Fourteen to eighteen (21\%); 19-30 (26\%); 31-50 (30\%); 51-65 (23\%).

Each person consented to a structured interview using the questionnaire based on the key themes that were identified in the qualitative phase of the Living \& Breathing Study. These were: attitudes towards asthma, perceptions of treatment, review perceptions, treatment and review preferences. The questionnaire was made up of 40 statements relating to each of these themes, and patients were asked which statement best applied to them. Patient views were then allocated to each theme.

\section{Results}

\section{Phase 1 (qualitative)}

Respondents expressed a high level of resignation and despondency concerning their condition and treatment. "You slot into a routine; you've got asthma, you get on with it; it's not going away; it's down to you" (female, 25-50 years old). This sense of resignation was most marked in older respondents and those who had had the condition from an early age: "I don't think about it. I just get on with it. It's just something that I've got' (female, 50-65 years old). There was also evidence that patients tended to de-prioritise their symptoms. "I've had this cough now the best part of 3 years. I always get a cough in the winter, so don't pay any attention to it' (male, 50-65 years old).

Many amongst the sample failed to take their preventer medication regularly. "I decided I was getting better and didn't want it anymore"' (female, 19-30 years old). Most respondents were devoted to their reliever inhaler. "I must admit, I wouldn't be without that one"' (female, 60-65 years old).

Respondents were very resistant to a treatment regime that was more complicated than their current two inhaler system. Ideally, patients preferred one inhaler. "If it is two or more inhalers then I don't want it"' (female, 19-30 years old). Respondents said that an easy to follow self-management plan would motivate them.

Many respondents gave a sceptical reaction to being shown management guidelines. "They [the guidelines] can't be right. My treatment doesn't do that"' (male, 25-50 years old).

For most respondents, asthma review was largely a functional interaction, and there was little evidence of an in-depth review process. "There's not much encouragement to become an even milder asthmatic" (male, 25-50); "At the end of the day, I want to be like I was with no treatment, when I could live my life free and I could go out and enjoy myself without panicking. Some doctors don't understand"' (male, 25-50).

Most respondents were disappointed with the approach to asthma care. "I haven't had my asthma looked at in 17 years (male, 25-50 years old). "I don't think there is enough trained personnel in asthma that you can get/in touch with" (mate, 25-50 years old).

ONurses rather than GPS were viewed as a more popular option for conducting asthma reviews because they were perceived as both accessible and friendly and more likely to talk about quality of life issues.

\section{Phase 2 (quantitative)}

Figs. 1 and 2 illustrate the age range and socioeconomic status of the 197 men and 320 women participating in the study.

Most patients acknowledged that asthma is a variable disease both in terms of time of day and

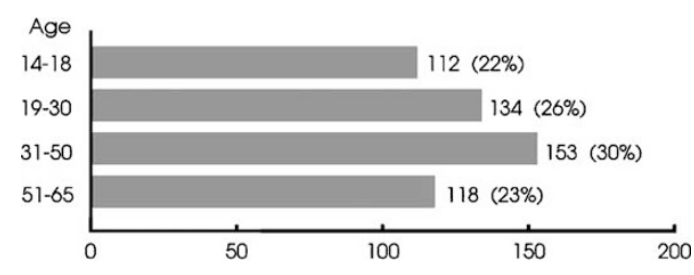

Figure 1 Age of respondents $(n=517)$. 


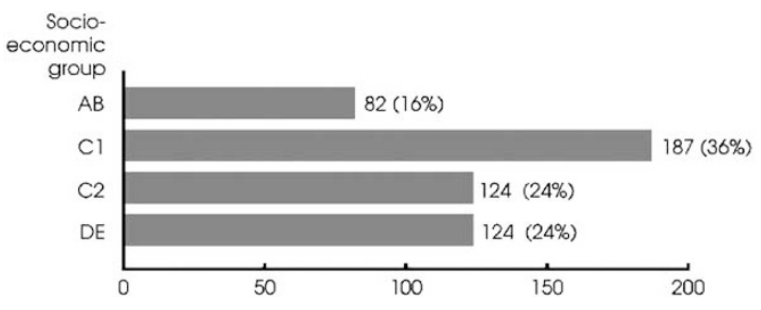

Figure 2 Socio-economic group $(n=517)$.

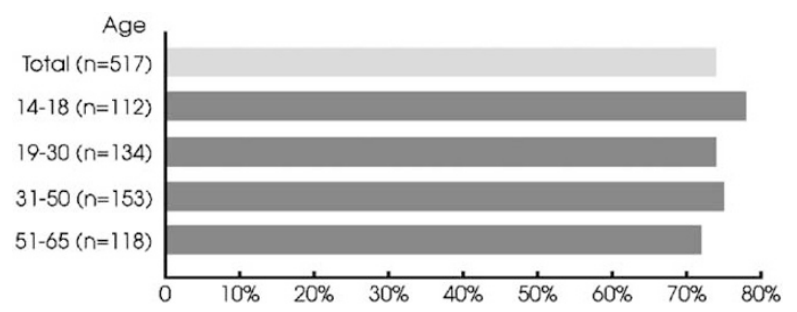

Figure 3 Percentage of respondents who said that their asthma varied in terms of severity at different times of the year, related to the age of the respondents.

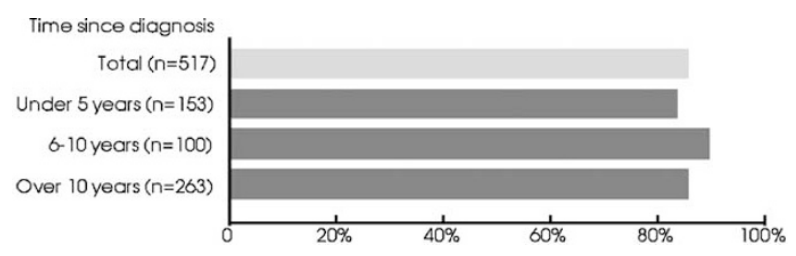

Figure 4 Percentage of respondents who said that their asthma varied in terms of severity at different times of the year, related to the time since diagnosis.

seasonal changes (Figs. 3 and 4 ). Forty percent $(n=206)$ responded to this variability by changing their own treatment regimes (Fig. 5); less than half $(n=222)$ did so on the advice of a healthcare professional. Indeed, when asthma worsened, $88 \%$ $(n=456)$ of respondents said they increased their medication: dose of their reliever inhaler (38\%; $n=199)$, preventer inhaler $(18 \% ; n=92)$ or both

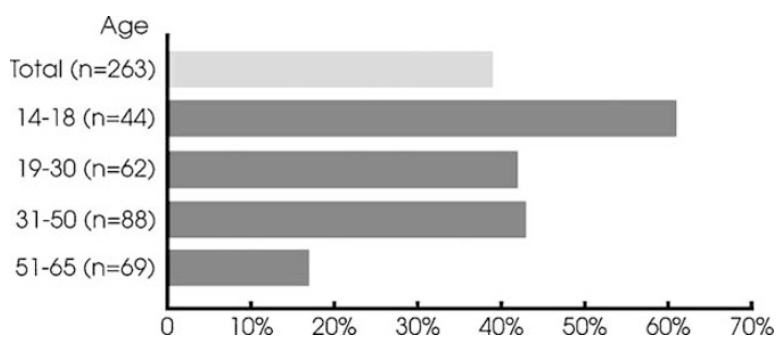

Figure 6 Percentage of respondents with poor preventer inhaler compliance.

inhalers $(32 \% ; n=165)$. Those with more complex treatment regimes were more likely to change their treatment $(63 \% ; n=42 / 67)$. Over a quarter $(27 \% ; n=142)$ changed their treatment based solely on their own experience and $43 \%(n=222)$ changed it following advice from a healthcare professional. Most respondents $(68 \% ; n=353)$ confirmed they would feel comfortable being able to adjust the dose of their inhaler without having to refer to a healthcare professional, and this number was consistent across age, sex, and socio-economic group.

Further review of compliance with treatment indicated that patient practice varied significantly, especially in relation to reliever medication. A third $(32 \% ; n=166)$ of respondents said they took a dose of their reliever inhaler every day, whether they needed it or not. And $11 \%(n=57)$ reported that they did not take any reliever medication, even when they experienced the onset of symptoms. In addition, 33\% $(n \in 173)$ of respondents reported missing a dose of their preventer inhaler; of these, $24 \%(n \neq 41)$ reported missing a dose $2-3$ days a week. Younger people with asthma seemed to be more non-compliant than older patients (Fig. 6).

Sixty-eight percent $(n=349)$ of respondents said they had never been provided with written information by their healthcare professional on

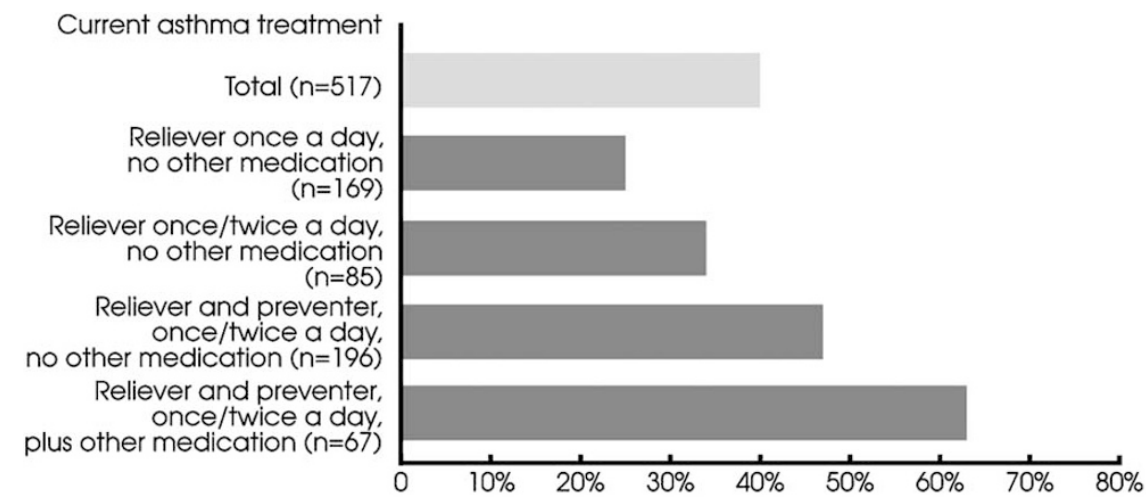

Figure 5 Percentage of respondents who said that they tend to change their own treatment regimen, related to current asthma therapy. 
how to take their prescribed medication. These patients also stated that they would feel comfortable being able to adjust the dose of their inhaler without having to refer to a healthcare professional.

Moreover, $80 \%(n=414)$ of respondents had never been provided with a personal, written, asthma action plan, which would outline how patients can adjust their prescribed medication depending on peak flow or symptoms [10].

Despite this, most patients recognised the need for education. Just over half $(55 \% ; n=284)$ confirmed they would find a personal, written, asthma action plan useful; $62 \%(n=323)$ confirmed they would feel comfortable following such a plan. Furthermore, $68 \%(n=353)$ said they felt comfortable adjusting their therapy.

Over half of those questioned $(62 \% ; n=319)$ felt it was very important to have their reliever inhaler close to hand, and $32 \%(n=166)$ used their reliever every day, whether they needed it or not. When asked how treatment regimes could be improved, $81 \%(n=418)$ said that a smaller number of inhalers to take was either appealing or very appealing, indicating that simplicity was regarded as key to improving asthma treatment regimes.

Patients had low expectations of how well they could be, i.e, of how well their asthma could be controlled. Around three-quarters of the sample $(n=394)$ felt that their asthma would not improve over time, with optimism about such improvement decreasing with increasing time since asthma di agnosis (Fig. 7). Most patients did not realise they could be better. Though $91 \%(n=468)$ felt their asthma was under control, $66 \%(n=339)$ reported they experienced symptoms at least two to three times a week (Fig. 8).

Indeed, before being shown the asthma symptom management goals outlined in the 'GINA' International guidelines [2] 58\% $(n=301)$ said they were very satisfied with the level of asthma care they

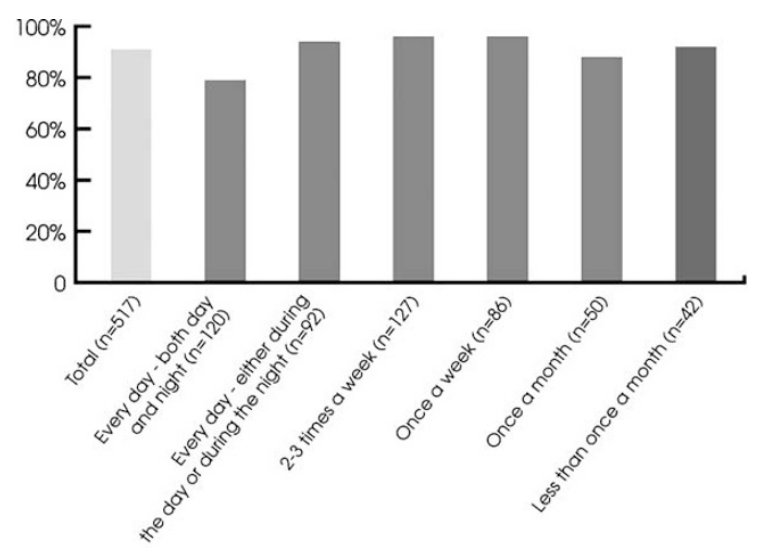

Figure 8 Perceived asthma control with varying frequency of asthma symptoms.

received. This dropped to $33 \%(n=173)$ after respondents were shown the symptom management goals.

A significant minority of patients was disillusioned or actively disappointed with the approach to asthma care. Fourteen percent $(n=74)$ of respondents said they had no on-going contact with a GP or other healthcare professional about their asthma. Almost half $(n=222)$ said they had no regular discussion with a GP or nurse and visited the GP surgery just to collect their repeat prescription. Three-quarters $(n=388)$ said they spoke to their doctor only when there was a problem, and only a third $(n=184)$ said they had an asthma trained

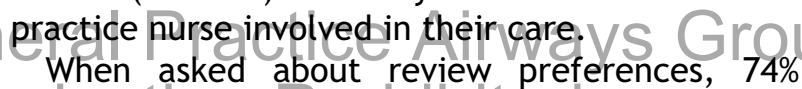
and 386$)$ said telephone access to asthma experts for information was either quite or very appealing.

And almost one-third (27\%; $n=67 / 252)$ ) of those with internet access said that e-mail access to an asthma expert was very appealing. Almost three-quarters of the sample $(71 \% ; n=366)$ said they had never been given advice on how their asthma could change in the future.

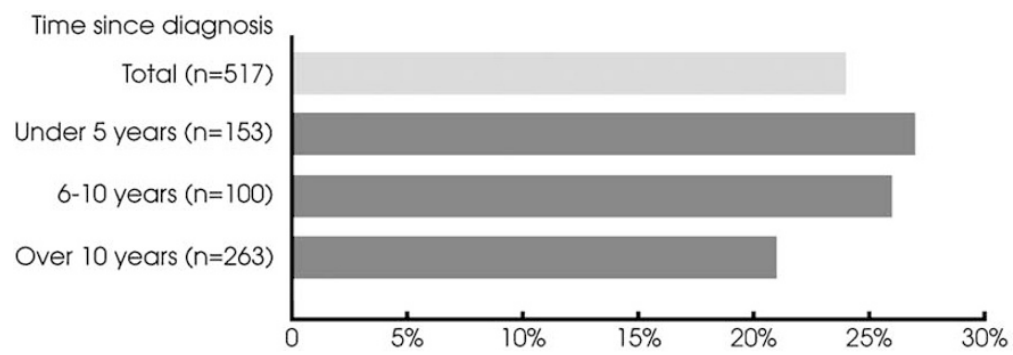

Figure 7 Percentage of patients who thought their asthma would improve over time, related to the time since diagnosis. 


\section{Discussion}

The Living \& Breathing Study gives some insight into patients' perceptions of their asthma; on what patients understand about their disease, what they think of its management and on their expectations for asthma control. It indicates that the majority are satisfied with the level of asthma care they receive, despite a high level of daily symptoms (two-thirds of respondents) and night-time symptoms (almost half of respondents). This finding is line with previous studies recording a high level of morbidity among asthma patients [1,4]. Most patients did not have high expectations that their disease could be managed in line with currently defined goals of treatment [2]. Around three-quarters of the sample felt their asthma would not improve over time, suggesting that patients with asthma accept daily symptoms as part of their condition, in spite of treatment, do not know they could be better and have very low expectations and aspirations of asthma management. This sense of resignation and despondency among patients concerning their asthma and its impact on their lives was most vividly revealed by the qualitative data. The finding that respondents' satisfaction with asthma care fell by almost half once they were shown the asthma symptom management goals outlined in the "GINA" international guidelines supports the observation that asthma patients do not realise they could be better. Most respondents were unaware of these goals and, indeed, expressed scepticism about them because their own experience did not match their expectations of them.

Unsurprisingly, given the high level of morbidity among the sample, $62 \%$ of respondents said it was "very important to keep their reliever medication close to hand", indicating over-use and/or over-dependence on this aspect of treatment (see further). Why patients have low expectations of what can be achieved with good asthma control may be, in part, explained by the finding that almost three-quarters of the respondents said they had never been given any advice on how their asthma might change in the future. A lack of education supporting patients to take a guided role in the management of their asthma was highlighted by the finding that a significant minority of patients did not conform to guidelines on the use of reliever medication. Indeed, as some patients took a daily dose of their reliever medication whether they needed it or not and some patients never took their reliever medication when they experienced symptoms it is not surprising that many patients were unable to relate to asthma symptom management goals.
A lack of encouragement among healthcare professionals to explore the reasons for persisting morbidity may lie at the heart of why patients appear so resigned to regular symptoms.

It is disappointing that almost half of the sample reported that they had no regular discussion with a healthcare professional about their asthma, indicating that regular review of asthma does not appear to be happening universally, despite the recommendation of asthma management guidelines that patients should receive education and regular medical review [8]. The fact that a large number spoke to their doctor only when they had a problem related to their asthma suggests that effective doctor-patient partnerships exploring asthma management goals to attain improved treatment and quality of life outcomes are extremely uncommon. Further support to the notion that poor communication between some patients and healthcare professionals is, at least, partly responsible for low patient expectations of asthma management is provided by the findings that almost a third of respondents changed their treatment based solely on their own experience and that a majority reported never having been provided with written information by their healthcare professional on how to take their prescribed medication further. However, this study provides evidence that many patients wish for a more constructive relationship with healthcare professionals and a more personalised approach to asthma management. Self-management education, incorporating the use of personal, written, asthma action plans, has been shown to improve healthoutcomes for people with asthma [7,9], and while our studrevealed a lowlevel of use of such plans, once they were explained there was a high level of patient support and acceptance of them. Our finding that more than $80 \%$ of respondents said they had never been provided with a plan but over half said they would find one helpful and feel comfortable using it contrasts with the findings of Jones and colleagues [10], who concluded that patients were not enthusiastic about the idea of using guided self-management plans for asthma management.

Our results are in line with those of a more recent study, which identified that asthma action plans were viewed positively by patients [11].

Patients are aware that their asthma will vary throughout the day and year, and that their medication can be adjusted accordingly. Despite such recognition, our study found that patients were not, in general, observed to step down their levels of medication in line with milder symptoms. This observation, coupled with the finding that poor compliance with preventer medication was commonplace, emphasises the need to provide 
appropriate education to patients so they can manage an appropriate reduction in steroid use.

There was a high level of enthusiasm for telephone access to asthma experts for information; suggesting that alternative methods of communication are acceptable and important to patients, and that patients would like more access to their own healthcare professional. This finding is in line with the results of Pinnock et al., who concluded that patients with asthma are satisfied with telephone consultations [12].

\section{Implications for practice}

Previous qualitative research has suggested that patients and healthcare professionals do not share the same treatment goals or lifestyle objectives $[1,3]$, underlining the need for healthcare professionals to adopt a flexible, patient-centred approach to asthma management. One aspect of such an approach may be to base asthma self-management on treating symptoms in terms of patients' ability, or lack of ability, to achieve their personal goals, rather than using objective lung function measurements, such as peak flow, to guide interventions [13]. Healthcare professionals may thus help to raise patient expectations of asthma management by discussing patient-defined goals, which could form the basis of a personal, written, asthma action plan, in the context of helping patients understand what can be achieved by good asthma control. Simplicity of management and treatment, for example through reducing the number of medications and inhalers, was identified as an important aspect of a more desirable management strategy by most respondents, and is another practical consideration for healthcare professionals aiming to adopt a more patient-centred approach to asthma care.

The consultation is an invaluable opportunity for patients and their doctors to negotiate desired outcomes and their preferred roles in achieving them. Although time constraints threaten successful patient-doctor partnerships, Jenkins et al. found that at least half of patients wanted to participate in decisions about treatment, and that consultations involving the patient in this way did not need to be long for outcomes to improve [14].

\section{Conclusion}

The findings from the Living \& Breathing Study indicate that there is a significant need to educate and support patients with asthma to enable them to form effective partnerships with healthcare profes- sionals so that more effective disease management occurs.

Our data further suggests that the introduction of a simplified approach to asthma management, based on patient-focused goals, is likely to be most successful. Implementing personal, written, asthma action plans is likely to raise low patient expectations about asthma treatment and help patients with asthma aspire to achieve their personal asthma goals. Such a management approach will help healthcare professionals support their patients more effectively and is in line with current best practice recommendations.

\section{Acknowledgements}

L\&B expert panel: Jack Barnes: Research Director, National Asthma Campaign; Monica Fletcher: Chief Executive Officer, National Asthma \& Respiratory Training Centre; Melinda Letts: Chair, Long-term Medical Conditions Alliance; David Price: GPIAG Professor of Primary Care Respiratory Medicine, Aberdeen; Darren Saunders: British Lung Foundation; Mike Stone: Director, The Patients Association; Liz Walker: Respiratory nurse specialist, Portsmouth; Association of Respiratory Nurse specialists; Lynn Young: Royal College of Nursing Community Health Adviser. The Living \& Breathing Study was sponsored by an educational grant from AstraZeneca UK Ltd.

\section{References}

O[1] Rabe kF, Vermeire PA, Soriano JB, Maier WC. Clinical management of asthma in 1999. The Asthma Insights and Realities in Europe (AIRE) study. Eur Respir J 2000;16:802-7.

[2] Global Initiative for Asthma. Bethesda, MD: National Institutes for Health, National Heart, Lung and Blood Institute. 1995 (revised 1998). Guidelines available from the GINA website http://www.ginasthma.com.

[3] Price D, Ryan D, Pearce L, Bride F. The AIR study: asthma in real life. Asthma J 1999;4:74-8.

[4] Smith NM. The Needs of People with Asthma survey and initial presentation of data. Asthma J 2000;5:133-7.

[5] Smith A, Partridge MR. Greater expectations. Asthma J 2000;5:106-7.

[6] Partridge MR, Hill S. On behalf of the 1998 World Asthma Meeting Education and Delivery of Care Working Group. Enhancing care for people with asthma: the role of communication, education, training and self-management. Eur Resp J 2000;16:333-48.

[7] Gibson PG, Coughlan J, Wilson AJ, Bauman A, Hensley MJ, Walters E. The effects of self-management education and regular practitioner review in adults with asthma (Cochrane Review). In: Cochrane Library, issue 3. Oxford: Update Software;1998.

[8] British Thoracic Society, Scottish Intercollegiate Guidelines Network. British guideline on the management of asthma. Thorax 2003; 58(Supplement 1):il-i94. 
[9] Lahdensuo A, Haahtela T, Herrala J, Kava T, Kiviranta $K$, Kuusisto $P$, et al. Randomised comparison of guided self-management and traditional treatment of asthma over one year. BMJ 1996;312:748-52.

[10] Jones A, Pill R, Adams S. Qualitative study of views of health professionals and patients on guided selfmanagement plans for asthma. BMJ 2000;321:150710.

[11] Douglass J, Aroni R, Goeman D, Stewart K, Sawyer S, Thien $F$, et al. A qualitative study of action plans for asthma. BMJ 2002;324:1003.
[12] Pinnock H, Bawden R, Proctor S, Wolfe S, Price D, Sheikh A. Accessibility, acceptability, and effectiveness in primary care of routine telephone review of asthma: pragmatic, randomised controlled trial. BMJ 2003;326:477.

[13] Turner M, Taylor D, Bennett R, Fitzgerald JM. A Randomised Trial Comparing Peak Expiratory Flow and Symptom Self-management Plans for Patients with Asthma Attending a Primary Care Clinic. Am J Respir Crit Care Med 1998;157:540-6.

[14] Jenkins L, Britten N, Barber N, Bradley CP, Stevenson FA. Consultations do not have to be longer. BMJ 2002;325:388.

\footnotetext{
Available online at www.sciencedirect.com

science $\boldsymbol{d}$ Direct.
}

\section{Copyright General Practice Airways Group Reproduction Prohibited}

